\title{
Some Discrete Nonlinear Inequalities in Two Variables
}

\author{
Baoju Sun (Corresponding author) \\ Department of Mathematics, Zhejiang Water Conservancy \& Hydropower University \\ Hangzhou 310018, China \\ E-mail: sunbj@zjwchc.com
}

\begin{abstract}
In this paper, some discrete nonlinear inequalities in two variables are established. The inequalities given here can be used as tools in the qualitative theory of certain finite difference equations.
\end{abstract}

Keywords: Discrete inequalities, Nonlinear, Two variables, Boundedness, Difference equation

\section{Introduction}

In the study of ordinary differential equations and integral equations one often deals with certain integral inequalities. The Gronwall-Bellman inequality and its various linear and nonlinear generalizations are crucial in the discussion of existence, uniqueness, continuation, boundedness, stability and other qualitative properties of solutions to differential and integral equations. The literature on such inequalities and their applications is vast; see Agarwal(2005), Bainov and Simeonov(1992), Cheung(2004, 2006), Cheung and Ren(2006), Dragomir and Kim ( 2003), Lipovan (2000), Pachpatte (2004a,b), Salem and Ralan (2004), and the references given therein. To handle difference equations, some discrete Gronwall-Bellman type inequalities are needed. During the past few years, some investigators have established some useful and interesting discrete Gronwall-Bellman type inequalities, see Cheung (2004, 2006), Cheung and Ren (2006), Salem and Ralan (2004). Cheung, W.S. (2004) had proved

Theorem A Suppose $u: \Omega \rightarrow \mathbb{R}_{+}$is a function on a 2-dimensional lattice $\Omega$, If $k \geq 0$ is constant and $a, b: \Omega \rightarrow \mathbb{R}_{+}, w \in$ $C\left(R_{+}, R_{+}\right)$are functions satisfying

(i) $w$ is nondecreasing with $w(r)>0$ for $r>0$; and

(ii) for any $(m, n) \in \Omega$,

$$
u^{2}(m, n) \leq k^{2}+\sum_{s=m_{0}}^{m-1} \sum_{t=n_{0}}^{n-1} a(s, t) u(s, t)+\sum_{s=m_{0}}^{m-1} \sum_{t=n_{0}}^{n-1} b(s, t) u(s, t) w(u(s, t)),
$$

then $u(m, n) \leq\left\{\Phi_{1}^{-1}\left[\Phi_{1}\left(k+A^{*}(m, n)\right)+B^{*}(m, n)\right]\right\}$ for all $(m, n) \in \Omega_{\left(m_{1}, n_{1}\right)}$, where

$$
A^{*}(m, n):=\sum_{s=m_{0}}^{m-1} \sum_{t=n_{0}}^{n-1} a(s, t), B^{*}(m, n):=\sum_{s=m_{0}}^{m-1} \sum_{t=n_{0}}^{n-1} b(s, t), \Phi_{1}(r):=\int_{1}^{r} \frac{d s}{w(s)},
$$

and $\left(m_{1}, n_{1}\right) \in \Omega$ is chosen such that $\Phi_{1}\left(k+A^{*}(m, n)\right)+B^{*}(m, n) \in \operatorname{Dom}\left(\Phi_{1}^{-1}\right)$ for all $(m, n) \in \Omega_{\left(m_{1}, n_{1}\right)}$. Very recently, Cheung and Ren (2006) established the following

Theorem B Suppose $u \in F_{+}(\Omega)$, If $c \geq 0, \alpha>0$ are constants and $b \in F_{+}(\Omega), \varphi \in C\left(R_{+}, R_{+}\right)$are functions satisfying

(i) $\varphi$ is nondecreasing with $\varphi(r)>0$ for $r>0$; and

(ii) for any $(m, n) \in \Omega$,

$$
u^{\alpha}(m, n) \leq c+\sum_{s=m_{0}}^{m-1} \sum_{t=n_{0}}^{n-1} b(s, t) \varphi(u(s, t))
$$

then

$$
u(m, n) \leq\left\{\Phi_{\alpha}^{-1}\left[\Phi_{\alpha}(c)+B^{*}(m, n)\right]\right\}^{1 / \alpha}
$$

for all $(m, n) \in \Omega_{\left(m_{1}, n_{1}\right)}$, where $B^{*}(m, n)$ is defined as in Theorem A, $\Phi_{\alpha}(r):=\int_{1}^{r} \frac{d s}{\varphi\left(s^{1 / \alpha}\right)}$, and $\left(m_{1}, n_{1}\right) \in \Omega$ is chosen such that $\Phi_{\alpha}(c)+B^{*}(m, n) \in \operatorname{Dom}\left(\Phi_{\alpha}^{-1}\right)$ for all $(m, n) \in \Omega_{\left(m_{1}, n_{1}\right)}$.

Motivated by the results in Cheung and Ren (2006), the main purpose of this paper is to establish some new discrete nonlinear inequalities in two variables. 
Throughout this paper, $I:=\left[m_{0}, M\right] \cap \mathbb{Z}$ and $J:=\left[n_{0}, N\right] \cap \mathbb{Z}$ are two fixed lattices of integral points in $\mathbb{R}$, where $m_{0}, n_{0} \in \mathbb{Z}, M, N \in \mathbb{Z} \cup\{\infty\}$. Let $\Omega:=I \times J \subset \mathbb{Z}^{2}, \mathbb{R}_{+}:=[0, \infty)$, and for any $(s, t) \in \Omega$,the sub-lattice $\left[m_{0}, s\right] \times\left[n_{0}, t\right] \cap \Omega$ of $\Omega$ will be denoted as $\Omega_{(s, t)}$. If $U$ is a lattice in $\mathbb{Z}$ (respectively $\mathbb{Z}^{2}$ ), the collection of all $\mathbb{R}$-valued functions on $U$ is denoted by $F(U)$,and that of all $\mathbb{R}_{+}$-valued funcctions by $F_{+}(U)$. For the sake of convenience, we extend the domain of definition of each function in $F(U)$ and $F_{+}(U)$ trivially to the ambient space $\mathbb{Z}$ (respectively $\mathbb{Z}^{2}$ ). So for example, a function in $F(U)$ is regarded as a function defined on $\mathbb{Z}$ (respectively $\mathbb{Z}^{2}$ ) with support in $U$. As usual, the collection of all continuous functions of a topological space $X$ into a topological space $Y$ will be denoted by $C(X, Y)$. If $U$ is a lattice in $\mathbb{Z}$, the difference operator $\Delta$ on $f \in F(\mathbb{Z})$ or $F_{+}(\mathbb{Z})$ is defined as $\Delta f(n):=f(n+1)-f(n), \quad n \in U$, and if $V$ is a lattice in $\mathbb{Z}^{2}$, the partial difference operators $\Delta_{1}$ and $\Delta_{2}$ on $u \in F\left(\mathbb{Z}^{2}\right)$ or $F_{+}\left(\mathbb{Z}^{2}\right)$ are defined as

$$
\begin{array}{ll}
\Delta_{1} u(m, n):=u(m+1, n)-u(m, n), & (m, n) \in V, \\
\Delta_{2} u(m, n):=u(m, n+1)-u(m, n), & (m, n) \in V .
\end{array}
$$

For any $\varphi, \psi \in C\left(\mathbb{R}_{+}, \mathbb{R}_{+}\right)$and any constant $\beta>0$, define

$$
\begin{gathered}
\Phi_{\beta}(r):=\int_{1}^{r} \frac{d s}{\varphi\left(s^{1 / \beta}\right)}, \quad \Psi_{\beta}(r):=\int_{1}^{r} \frac{d s}{\psi\left(s^{1 / \beta}\right)}, \quad r>0, \\
\Phi_{\beta}(0):=\lim _{r \rightarrow 0^{+}} \Phi_{\beta}(r), \quad \Psi_{\beta}(0):=\lim _{r \rightarrow 0^{+}} \Psi_{\beta}(r) .
\end{gathered}
$$

Note that we allow $\Phi_{\beta}(0)$ and $\Psi_{\beta}(0)$ to be $-\infty$ here.

\section{Main results}

Theorem 2.1. Suppose $u \in F_{+}(\Omega)$, If $c \geq 0, \alpha>0$ are constants and $b, h \in F_{+}(\Omega), \varphi \in C\left(R_{+}, R_{+}\right)$are functions satisfying

(i) $\varphi$ is nondecreasing with $\varphi(r)>0$ for $r>0$; and

(ii) for any $(m, n) \in \Omega$,

$$
u^{\alpha}(m, n) \leq c+\sum_{s=m_{0}}^{m-1} \sum_{t=n_{0}}^{n-1} b(s, t)\left(\sum_{\tau=m_{0}}^{s} \sum_{\sigma=n_{0}}^{t} h(\tau, \sigma) \varphi(u(\tau, \sigma))\right)
$$

Then

$$
u(m, n) \leq\left\{\Phi_{\alpha}^{-1}\left[\Phi_{\alpha}(c)+B(m, n)\right]\right\}^{1 / \alpha}
$$

for all $(m, n) \in \Omega_{\left(m_{1}, n_{1}\right)}$, where

$$
B(m, n):=\sum_{s=m_{0}}^{m-1} \sum_{t=n_{0}}^{n-1} b(s, t)\left(\sum_{\tau=m_{0}}^{s} \sum_{\sigma=n_{0}}^{t} h(\tau, \sigma)\right)
$$

$\Phi_{\alpha}^{-1}$ is the inverse of $\Phi_{\alpha}$, and $\left(m_{1}, n_{1}\right) \in \Omega$ is chosen such that $\Phi_{\alpha}(c)+B(m, n) \in \operatorname{Dom}\left(\Phi_{\alpha}^{-1}\right)$ for all $(m, n) \in \Omega_{\left(m_{1}, n_{1}\right)}$.

Proof. It suffices to consider the case $c>0$, for the case $c=0$ can be arrived at by continuity argument. Denote by $g(m, n)$ the right-hand side of (1). Then $g>0, u \leq g^{1 / \alpha}$ on $\Omega$, and $g$ is nondecreasing in each variable. Hence for any $(m, n) \in \Omega$,

$$
\begin{aligned}
\Delta_{1} g(m, n) & =g(m+1, n)-g(m, n)=\sum_{t=n_{0}}^{n-1} b(m, t)\left(\sum_{\tau=m_{0}}^{m} \sum_{\sigma=n_{0}}^{t} h(\tau, \sigma) \varphi(u(\tau, \sigma))\right) \\
\leq & \sum_{t=n_{0}}^{n-1} b(m, t)\left(\sum_{\tau=m_{0}}^{m} \sum_{\sigma=n_{0}}^{t} h(\tau, \sigma) \varphi\left(g^{1 / \alpha}(\tau, \sigma)\right)\right) \leq \varphi\left(g^{1 / \alpha}(m, n-1)\right) \sum_{t=n_{0}}^{n-1} b(m, t)\left(\sum_{\tau=m_{0}}^{m} \sum_{\sigma=n_{0}}^{t} h(\tau, \sigma)\right) .
\end{aligned}
$$

by (4),

$$
\begin{aligned}
\Delta_{1}\left(\Phi_{\alpha} \circ g\right)(m, n) & =\Phi_{\alpha}(g(m+1, n))-\Phi_{\alpha}(g(m, n))=\int_{g(m, n)}^{g(m+1, n)} \frac{1}{\varphi\left(s^{1 / \alpha}\right)} d s \\
& \leq \frac{1}{\varphi\left(g^{1 / \alpha}(m, n)\right)} \Delta_{1} g(m, n) \leq \frac{\varphi\left(g^{1 / \alpha}(m, n-1)\right)}{\varphi\left(g^{1 / \alpha}(m, n)\right)} \sum_{t=n_{0}}^{n-1} b(m, t)\left(\sum_{\tau=m_{0}}^{m} \sum_{\sigma=n_{0}}^{t} h(\tau, \sigma)\right) \\
& \leq \sum_{t=n_{0}}^{n-1} b(m, t)\left(\sum_{\tau=m_{0}}^{m} \sum_{\sigma=n_{0}}^{t} h(\tau, \sigma)\right)
\end{aligned}
$$


for all $(m, n) \in \Omega$. Therefore

$$
\sum_{s=m_{0}}^{m-1} \Delta_{1}\left(\Phi_{\alpha} \circ g\right)(s, n) \leq \sum_{s=m_{0}}^{m-1} \sum_{t=n_{0}}^{n-1} b(s, t)\left(\sum_{\tau=m_{0}}^{s} \sum_{\sigma=n_{0}}^{t} h(\tau, \sigma)\right)=B(m, n) .
$$

On the other hand, it is elementary to check that

$$
\sum_{s=m_{0}}^{m-1} \Delta_{1}\left(\Phi_{\alpha} \circ g\right)(s, n)=\Phi_{\alpha} \circ g(m, n)-\Phi_{\alpha} \circ g\left(m_{0}, n\right),
$$

thus

$$
\Phi_{\alpha} \circ g(m, n) \leq \Phi_{\alpha} \circ g\left(m_{0}, n\right)+B(m, n)=\Phi_{\alpha}(c)+B(m, n) .
$$

Since $\Phi_{\alpha}^{-1}$ is increasing on Dom $\Phi_{\alpha}^{-1}$, this yields

$$
g(m, n) \leq \Phi_{\alpha}^{-1}\left[\Phi_{\alpha}(c)+B(m, n)\right]
$$

for all $(m, n) \in \Omega_{\left(m_{1}, n_{1}\right)}$.

Using (6) in $u \leq g^{1 / \alpha}$, we have the required inequality in (2). This completes the proof of Theorem 2.1.

\section{Remarks 1.}

(i) When $h(\tau, \sigma)=\left\{\begin{array}{ll}1, & (\tau, \sigma)=(s, t) \\ 0, & (\tau, \sigma) \neq(s, t)\end{array}\right.$, Theorem 2.1 reduces to Theorem $\mathrm{B}$.

(ii) In many cases the nondecreasing function $\varphi$ satisfies $\int_{1}^{\infty} \frac{1}{\varphi\left(s^{1 / \alpha}\right)} d s=\infty$.

For example, $\varphi=$ constant $>0, \varphi(r)=r^{\alpha}, \varphi(r)=r^{\alpha} \ln \left(1+r^{\alpha}\right)$,etc., are such functions (see Constantin, A. (1995)). In such cases $\Phi_{\alpha}(\infty)=\infty$ and so we may take $m_{1}=M, n_{1}=N$. In particular, inequality (2) holds for all $(m, n) \in \Omega$.

Theorem 2.2. Suppose $u \in F_{+}(\Omega)$, If $k \geq 0, p>1$ are constants and $a, b, h \in F_{+}(\Omega), \varphi \in C\left(R_{+}, R_{+}\right)$are functions satisfying

(i) $\varphi$ is nondecreasing with $\varphi(r)>0$ for $r>0$; and

(ii) for any $(m, n) \in \Omega$,

$$
u^{p}(m, n) \leq k+\sum_{s=m_{0}}^{m-1} \sum_{t=n_{0}}^{n-1} a(s, t) u(s, t)+\sum_{s=m_{0}}^{m-1} \sum_{t=n_{0}}^{n-1} b(s, t) u(s, t)\left(\sum_{\tau=m_{0}}^{s} \sum_{\sigma=n_{0}}^{t} h(\tau, \sigma) \varphi(u(\tau, \sigma))\right)
$$

Then

$$
u(m, n) \leq\left\{\Phi_{p-1}^{-1}\left[\Phi_{p-1}\left(k^{1-1 / p}+A(m, n)\right)+B(m, n)\right]\right\}^{1 /(p-1)}
$$

for all $(m, n) \in \Omega_{\left(m_{1}, n_{1}\right)}$, where

$$
A(m, n):=\sum_{s=m_{0}}^{m-1} \sum_{t=n_{0}}^{n-1} a(s, t), B(m, n):=\sum_{s=m_{0}}^{m-1} \sum_{t=n_{0}}^{n-1} b(s, t)\left(\sum_{\tau=m_{0}}^{s} \sum_{\sigma=n_{0}}^{t} h(\tau, \sigma)\right),
$$

and $\left(m_{1}, n_{1}\right) \in \Omega$ is chosen such that $\Phi_{p-1}\left(k^{1-1 / p}+A(m, n)\right)+B(m, n) \in \operatorname{Dom}\left(\Phi_{p-1}^{-1}\right)$ for all $(m, n) \in \Omega_{\left(m_{1}, n_{1}\right)}$.

Proof. Similar to the proof of Theorem 2.1, it suffices to consider the case $k>0$, for the case $k=0$ can be arrived at by continuity argument. Denote by $f(m, n)$ the right-hand side of (7). Then $f>0, u \leq f^{1 / p}$ on $\Omega$, and $f$ is nondecreasing in each variable. Hence for any $(m, n) \in \Omega$, 


$$
\begin{aligned}
\Delta_{1} f(m, n) & =f(m+1, n)-f(m, n) \\
& =\sum_{t=n_{0}}^{n-1} a(m, t) u(m, t)+\sum_{t=n_{0}}^{n-1} b(m, t) u(m, t)\left(\sum_{\tau=m_{0}}^{m} \sum_{\sigma=n_{0}}^{t} h(\tau, \sigma) \varphi(u(\tau, \sigma))\right) \\
& \leq \sum_{t=n_{0}}^{n-1} a(m, t) f^{1 / p}(m, t)+\sum_{t=n_{0}}^{n-1} b(m, t) f^{1 / p}(m, t)\left(\sum_{\tau=m_{0}}^{m} \sum_{\sigma=n_{0}}^{t} h(\tau, \sigma) \varphi\left(f^{1 / p}(\tau, \sigma)\right)\right) \\
& \leq f^{1 / p}(m, n-1)\left[\sum_{t=n_{0}}^{n-1} a(m, t)+\sum_{t=n_{0}}^{n-1} b(m, t)\left(\sum_{\tau=m_{0}}^{m} \sum_{\sigma=n_{0}}^{t} h(\tau, \sigma) \varphi\left(f^{1 / p}(\tau, \sigma)\right)\right)\right]
\end{aligned}
$$

$\mathrm{Or}$

$$
\frac{\Delta_{1} f(m, n)}{f^{1 / p}(m, n-1)} \leq \sum_{t=n_{0}}^{n-1} a(m, t)+\sum_{t=n_{0}}^{n-1} b(m, t)\left(\sum_{\tau=m_{0}}^{m} \sum_{\sigma=n_{0}}^{t} h(\tau, \sigma) \varphi\left(f^{1 / p}(\tau, \sigma)\right)\right)
$$

Therefor, for any $(m, n) \in \Omega$,

$$
\begin{aligned}
\sum_{s=m_{0}}^{m-1} \frac{\Delta_{1} f(s, n)}{f^{1 / p}(s, n-1)} & \leq \sum_{s=m_{0}}^{m-1} \sum_{t=n_{0}}^{n-1} a(s, t)+\sum_{s=m_{0}}^{m-1} \sum_{t=n_{0}}^{n-1} b(s, t)\left(\sum_{\tau=m_{0}}^{s} \sum_{\sigma=n_{0}}^{t} h(\tau, \sigma) \varphi\left(f^{1 / p}(\tau, \sigma)\right)\right) \\
& =A(m, n)+\sum_{s=m_{0}}^{m-1} \sum_{t=n_{0}}^{n-1} b(s, t)\left(\sum_{\tau=m_{0}}^{s} \sum_{\sigma=n_{0}}^{t} h(\tau, \sigma) \varphi\left(f^{1 / p}(\tau, \sigma)\right)\right) .
\end{aligned}
$$

On the other hand, by the nondecreasing property of $f$ in each variable, we have

$$
\begin{aligned}
& \sum_{s=m_{0}}^{m-1} \frac{\Delta_{1} f(s, n)}{f^{1 / p}(s, n-1)} \\
= & \frac{f(m, n)}{f^{1 / p}(m-1, n-1)}-\frac{f(m-1, n)}{f^{1 / p}(m-1, n-1)}+\frac{f(m-1, n)}{f^{1 / p}(m-2, n-1)}-\frac{f(m-2, n)}{f^{1 / p}(m-2, n-1)}+\ldots \\
& +\frac{f\left(m_{0}+1, n\right)}{f^{1 / p}\left(m_{0}, n-1\right)}-\frac{f\left(m_{0}, n\right)}{f^{1 / p}\left(m_{0}, n-1\right)} \\
= & \frac{f(m, n)}{f^{1 / p}(m-1, n-1)}+\sum_{s=1}^{m-m_{0}-1} f(m-s, n)\left[\frac{1}{f^{1 / p}(m-s-1, n-1)}-\frac{1}{f^{1 / p}(m-s, n-1)}\right]-\frac{f\left(m_{0}, n\right)}{f^{1 / p}\left(m_{0}, n-1\right)} \\
\geq & \frac{f(m, n)}{f^{1 / p}(m-1, n-1)}-\frac{f\left(m_{0}, n\right)}{f^{1 / p}\left(m_{0}, n-1\right)} \\
= & \frac{f(m, n)}{f^{1 / p}(m-1, n-1)}-k^{1-\frac{1}{p}} \geq f^{1-\frac{1}{p}}(m, n)-k^{1-\frac{1}{p}}
\end{aligned}
$$

for any $(m, n) \in \Omega$. Hence we obtain

$f^{1-\frac{1}{p}}(m, n) \leq k^{1-\frac{1}{p}}+A(m, n)+\sum_{s=m_{0}}^{m-1} \sum_{t=n_{0}}^{n-1} b(s, t)\left(\sum_{\tau=m_{0}}^{s} \sum_{\sigma=n_{0}}^{t} h(\tau, \sigma) \varphi\left(f^{1 / p}(\tau, \sigma)\right)\right)$

for any $(m, n) \in \Omega$. In particular, since $A(m, n)$ is nondecreasing in each variable, for any fixed $(\bar{m}, \bar{n}) \in \Omega_{\left(m_{1}, n_{1}\right)}$, $f^{1-\frac{1}{p}}(m, n)=\left[f^{\frac{1}{p}}(m, n)\right]^{p-1} \leq\left(k^{1-\frac{1}{p}}+A(\bar{m}, \bar{n})\right)+\sum_{s=m_{0}}^{m-1} \sum_{t=n_{0}}^{n-1} b(s, t)\left(\sum_{\tau=m_{0}}^{s} \sum_{\sigma=n_{0}}^{t} h(\tau, \sigma) \varphi\left(f^{1 / p}(\tau, \sigma)\right)\right)$

for all $(m, n) \in \Omega_{(\bar{m}, \bar{n})}$. Now by applying Theorem 2.1 to the function $f^{\frac{1}{p}}(m, n)$, we have

$$
u(m, n) \leq f^{\frac{1}{p}}(m, n) \leq\left\{\Phi_{p-1}^{-1}\left[\Phi_{p-1}\left(k^{1-1 / p}+A(\bar{m}, \bar{n})\right)+B(m, n)\right]\right\}^{1 /(p-1)}
$$

for all $(m, n) \in \Omega_{(\bar{m}, \bar{n})}$. In particular, this gives 


$$
u(\bar{m}, \bar{n}) \leq\left\{\Phi_{p-1}^{-1}\left[\Phi_{p-1}\left(k^{1-1 / p}+A(\bar{m}, \bar{n})\right)+B(\bar{m}, \bar{n})\right]\right\}^{1 /(p-1)}
$$

Since $(\bar{m}, \bar{n}) \in \Omega_{\left(m_{1}, n_{1}\right)}$ is arbitrary, this concludes the proof of the theorem.

\section{Remarks 2.}

(i)When $p=2, h(\tau, \sigma)=\left\{\begin{array}{ll}1, & (\tau, \sigma)=(s, t) \\ 0, & (\tau, \sigma) \neq(s, t)\end{array}\right.$, Theorem 2.2 reduces to Theorem A.

(ii) Similar to the previous remark, in mamy cases $\Phi_{p-1}(\infty)=\infty$ and so in these situations, inequality (8) holds for all $(m, n) \in \Omega$.

Theorem 2.2 can easily be applied to generate other useful discrete inequalities in more general situations. For example, we have

Theorem 2.3. Suppose $u \in F_{+}(\Omega)$, If $k \geq 0, p>q>0$ are constants and $a, b, h \in F_{+}(\Omega), \varphi \in C\left(R_{+}, R_{+}\right)$are functions satisfying

(i) $\varphi$ is nondecreasing with $\varphi(r)>0$ for $r>0$; and

(ii) for any $(m, n) \in \Omega$,

$$
u^{p}(m, n) \leq k+\sum_{s=m_{0}}^{m-1} \sum_{t=n_{0}}^{n-1} a(s, t) u^{q}(s, t)+\sum_{s=m_{0}}^{m-1} \sum_{t=n_{0}}^{n-1} b(s, t) u^{q}(s, t)\left(\sum_{\tau=m_{0}}^{s} \sum_{\sigma=n_{0}}^{t} h(\tau, \sigma) \varphi(u(\tau, \sigma))\right)
$$

then

$$
u(m, n) \leq\left\{\Phi_{p-q}^{-1}\left[\Phi_{p-q}\left(k^{1-q / p}+A(m, n)\right)+B(m, n)\right]\right\}^{1 /(p-q)}
$$

for all $(m, n) \in \Omega_{\left(m_{1}, n_{1}\right)}$, where $A(m, n), B(m, n)$ are defined as in Theorem 2.2, and $\left(m_{1}, n_{1}\right) \in \Omega$ is chosen such that $\Phi_{p-q}\left(k^{1-q / p}+A(m, n)\right)+B(m, n) \in \operatorname{Dom}\left(\Phi_{p-q}^{-1}\right)$ for all $(m, n) \in \Omega_{\left(m_{1}, n_{1}\right)}$.

Proof. For any $r>0$, define

$$
\psi(r)=\varphi\left(r^{1 / q}\right)
$$

Then clearly $\psi$ satisfies condition (i) of Theorem 2.2. By (12)

$$
u^{p}(m, n) \leq k+\sum_{s=m_{0}}^{m-1} \sum_{t=n_{0}}^{n-1} a(s, t) u^{q}(s, t)+\sum_{s=m_{0}}^{m-1} \sum_{t=n_{0}}^{n-1} b(s, t) u^{q}(s, t)\left(\sum_{\tau=m_{0}}^{s} \sum_{\sigma=n_{0}}^{t} h(\tau, \sigma) \psi\left(u^{q}(\tau, \sigma)\right)\right)
$$

for any $(m, n) \in \Omega$.

Writing $v=u^{q}$, this becomes

$$
v^{\frac{p}{q}}(m, n) \leq k+\sum_{s=m_{0}}^{m-1} \sum_{t=n_{0}}^{n-1} a(s, t) v(s, t)+\sum_{s=m_{0}}^{m-1} \sum_{t=n_{0}}^{n-1} b(s, t) v(s, t)\left(\sum_{\tau=m_{0}}^{s} \sum_{\sigma=n_{0}}^{t} h(\tau, \sigma) \psi(v(\tau, \sigma))\right)
$$

Since $\frac{p}{q}>1$, it follows from Theorem 2.2 that

$$
v(m, n) \leq\left\{\Psi_{p / q-1}^{-1}\left[\Psi_{p / q-1}\left(k^{1-q / p}+A(m, n)\right)+B(m, n)\right]\right\}^{1 /(p / q-1)}=\left\{\Psi_{(p-q) / q}^{-1}\left[\Psi_{(p-q) / q}\left(k^{1-q / p}+A(m, n)\right)+B(m, n)\right]\right\}^{\frac{q}{p-q}}
$$

for all $(m, n) \in \Omega_{\left(m_{1}, n_{1}\right)}$. Now it is elementary to check by the definition $\psi$ in (14) that $\Psi_{\frac{p-q}{q}}(r)=\Phi_{p-q}(r)$, thus we have

$$
v(m, n) \leq\left\{\Phi_{p-q}^{-1}\left[\Phi_{p-q}\left(k^{1-q / p}+A(m, n)\right)+B(m, n)\right]\right\}^{q /(p-q)}
$$

for all $(m, n) \in \Omega_{\left(m_{1}, n_{1}\right)}$, or

$$
u(m, n)=v^{\frac{1}{q}}(m, n) \leq\left\{\Phi_{p-q}^{-1}\left[\Phi_{p-q}\left(k^{1-q / p}+A(m, n)\right)+B(m, n)\right]\right\}^{1 /(p-q)}
$$


for all $(m, n) \in \Omega_{\left(m_{1}, n_{1}\right)}$, where $\left(m_{1}, n_{1}\right) \in \Omega$ is chosen such that $\Phi_{p-q}\left(k^{1-q / p}+A(m, n)\right)+B(m, n) \in \operatorname{Dom}\left(\Phi_{p-q}^{-1}\right)$ for all $(m, n) \in \Omega_{\left(m_{1}, n_{1}\right)}$.

An important special case of Theorem 2.3 is the following

Corollary 2.4. Suppose $u \in F_{+}(\Omega)$, If $k \geq 0, p>1$ are constants and $a, b, h \in F_{+}(\Omega), \varphi \in C\left(R_{+}, R_{+}\right)$are functions satisfying

(i) $\varphi$ is nondecreasing with $\varphi(r)>0$ for $r>0$; and

(ii) for any $(m, n) \in \Omega$,

$$
u^{p}(m, n) \leq k+\sum_{s=m_{0}}^{m-1} \sum_{t=n_{0}}^{n-1} a(s, t) u^{p-1}(s, t)+\sum_{s=m_{0}}^{m-1} \sum_{t=n_{0}}^{n-1} b(s, t) u^{p-1}(s, t)\left(\sum_{\tau=m_{0}}^{s} \sum_{\sigma=n_{0}}^{t} h(\tau, \sigma) \varphi(u(\tau, \sigma))\right.
$$

Then

$$
u(m, n) \leq \Phi_{1}^{-1}\left[\Phi_{1}\left(k^{\frac{1}{p}}+A(m, n)\right)+B(m, n)\right]
$$

for all $(m, n) \in \Omega_{\left(m_{1}, n_{1}\right)}$, where $A(m, n), B(m, n)$ are defined as in Theorem 2.2, and $\left(m_{1}, n_{1}\right) \in \Omega$ is chosen such that $\Phi_{1}\left(k^{1 / p}+A(m, n)\right)+B(m, n) \in \operatorname{Dom}\left(\Phi_{1}^{-1}\right)$ for all $(m, n) \in \Omega_{\left(m_{1}, n_{1}\right)}$.

In particular, we have the following useful consequence.

Corollary 2.5. Suppose $u \in F_{+}(\Omega)$, If $k \geq 0, p>1$ are constants and $a, b, h \in F_{+}(\Omega)$ are functions such that for any $(m, n) \in \Omega$,

$$
u^{p}(m, n) \leq k+\sum_{s=m_{0}}^{m-1} \sum_{t=n_{0}}^{n-1} a(s, t) u^{p-1}(s, t)+\sum_{s=m_{0}}^{m-1} \sum_{t=n_{0}}^{n-1} b(s, t) u^{p-1}(s, t)\left(\sum_{\tau=m_{0}}^{s} \sum_{\sigma=n_{0}}^{t} h(\tau, \sigma) u(\tau, \sigma)\right),
$$

then $u(m, n) \leq\left(k^{\frac{1}{p}}+A(m, n)\right) \exp (B(m, n))$ for all $(m, n) \in \Omega$, where $A(m, n), B(m, n)$ are defined as in Theorem 2.2 .

\section{Application}

Consider the following difference equation

$$
\begin{aligned}
\Delta_{12} z^{p}(m, n)= & z^{p-1}(m, n) a(m, n) \\
& +z^{p-1}(m, n) M\left(m, n, z(m, n), \sum_{\tau=m_{0}}^{m} \sum_{\sigma=n_{0}}^{n} N(\tau, \sigma, z(\tau, \sigma))\right)
\end{aligned}
$$

Satisfying

$$
z\left(m, n_{0}\right)=f(m), z\left(m_{0}, n\right)=g(n), f\left(m_{0}\right)=g\left(n_{0}\right)=0 .
$$

Where $p>1, a \in F_{+}(\Omega)$ are given.

The following theorem deals with the estimate on the solution of problem (??)-(??).

Theorem 3.1. Suppose that

$$
\begin{gathered}
M(p, q, j, k) \leq b(p, q)|k|, \\
N(p, q, k) \leq h(p, q) \varphi(|k|), \\
|f(m)|^{p}+|g(n)|^{p} \leq k \quad \text { for some } k \geq 0,(m, n) \in \Omega
\end{gathered}
$$

Where $b, h \in F_{+}(\Omega), \varphi, \Phi_{1}$ are as defined in corollary 2.4 .

If $z(m, n)$ is any solution of (17)-(18), then

$$
|z(m, n)| \leq \Phi_{1}^{-1}\left[\Phi_{1}\left(k^{\frac{1}{p}}+A(m, n)\right)+B(m, n)\right] .
$$


Proof. It is easy to see that the solution $z(m, n)$ of the problem (17)-(18) satisfies the equivalent equation

$$
\begin{aligned}
z^{p}(m, n)= & f^{p}(m)+g^{p}(n)+\sum_{s=m_{0}}^{m-1} \sum_{t=n_{0}}^{n-1} z^{p-1}(s, t) a(s, t) \\
& +\sum_{s=m_{0}}^{m-1} \sum_{t=n_{0}}^{n-1} z^{p-1}(s, t) M\left(s, t, z(s, t), \sum_{\tau=m_{0}}^{s} \sum_{\sigma=n_{0}}^{t} N(\tau, \sigma, z(\tau, \sigma))\right)
\end{aligned}
$$

From (23), (18), (19), (20), (21), we have

$$
\begin{aligned}
|z(m, n)|^{p} \leq & k+\sum_{s=m_{0}}^{m-1} \sum_{t=n_{0}}^{n-1} a(s, t)|z(s, t)|^{p-1} \\
& +\sum_{s=m_{0}}^{m-1} \sum_{t=n_{0}}^{n-1} b(s, t)|z(s, t)|^{p-1}\left(\sum_{\tau=m_{0}}^{s} \sum_{\sigma=n_{0}}^{t} h(\tau, \sigma) \varphi(|z(s, t)|)\right)
\end{aligned}
$$

Now a suitable application of the inequality (16) given in corollary 2.4 to (24) yields (22).

\section{References}

Agarwal, R. P., Deng, S. and Zhang, W. (2005). Generalization of a retarded Gronwall-like inequality and its applications. Appl. Math. Comput., 165, 599-612.

Bainov, D. and Simeonov P. (1992). Integral Inequalities and Applications, Kluwer Academic, Dordrecht.

Constantin, A. (1995). Global solutions of perturbed differential equations [French], C. R.Acad. Sci. Paris, 320, 13191322.

Cheung, W. S. (2006). Some new nonlinear inequalities and applications to boundary value problems, Nonlinear Anal., 64, 2112-2128.

Cheung, W. S. (2004). Some discrete nonlinear inequalities and applications to boundary value problems for difference equations. J. Differ. Equat. Appl., 10, 213-223.

Cheung, W. S. and Ren, J. L. (2006). Discrete non-linear inequalities and applications to boundary value problems. J. Math. Anal. Appl. , 319, 708-724.

Dragomir, S. S. and Kim, Y. H. (2003). Some integral inequalities for functions of two variables. Electr. J. Diff. Equa., $10,1-13$.

Lipovan, O. (2000). A retarded Gronwall-like inequality and its applications. J. Math. Anal. Appl., 252, 389-401.

Pachpatte, B. G. (2004a). On a certain retarded integral inequality and applications. J. Inequal. Pure Appl. Math., 5(1), article 19.

Pachpatte, B. G. (2004b). On some new nonlinear retarded integral inequalities. J. Inequal. Pure Appl. Math., 5(3), article 80 .

Salem, Sh. and Ralan, K. R. (2004). Some new discrete inequalities and their applications. J. Inequal. Pure Appl. Math, $5(1)$, article 2. 